\title{
Sistema Produto+Serviço para o cozinhar sustentável: Estudo de Caso Projeto Monno
}

\author{
Product+Service System for sustainable cooking: Case Study Project Monno
}

\author{
COSTA JUNIOR., Jairo da \\ Mestrando; Universidade Federal do Paraná \\ jairo.costa.junior@gmail.com \\ PEREIRA, João Victor Inácio \\ Mestre; Universidade Federal do Paraná \\ joao.victor.inacio@gmail.com \\ ZACAR, Cláudia H. \\ Mestre; Universidade Federal do Paraná \\ claudiazacar@yahoo.com.br \\ ENRICONI, Alessandra \\ Graduanda; Universidade Federal do Paraná \\ amenriconi@gmail.com \\ GOMES, Nivaldo Simões; \\ Mestrando; Universidade Federal do Paraná \\ nivaldo_gomes@yahoo.com \\ SANTOS, Aguinaldo dos \\ PhD; Universidade Federal do Paraná \\ asantos@ufpr.br
}

\begin{abstract}
Resumo
Diante das crises ambientais, econômicas e sócio-culturais fica patente a necessidade de rever os atuais padrões de produção, distribuição e consumo de alimentos. No presente estudo utiliza-se o método MEPSS para o desenvolvimento de um Sistema Produto+Serviço (PSS) com o objetivo de gerar soluções sistêmicas para o ato de cozinhar sustentável. Como resultado, a solução proposta reduz o conteúdo material necessário para o atendimento da unidade de satisfação. A expansão do campo de atuação do design traz novas oportunidades de desenvolvimento de soluções sistêmicas, destacando o estudo do PSS como um meio prático de atingir melhores resultados projetuais.
\end{abstract}

Palavras Chave: Design; Sistema produto+serviço; Cozinhar sustentável.

\begin{abstract}
In the face of environmental, economic and socio-cultural crises is evident the necessity of revise the existing patterns of production, distribution and consumption in the food sector. This study aims to present the benefits of Methodology of Product Service System (MEPSS) in order to generate systemic solutions to the act of cooking in a sustainable way. As a result, the proposed solution reduces the material content needed to satisfy customer needs competitively and with lower environmental impact over the life cycle. The expansion of the field of design brings new opportunities to develop systemic solutions, emphasizing the study of PSS as a practical means to achieve better projects results.
\end{abstract}

Keywords: Design; Product service system; Sustainable cooking. 


\section{Introdução}

Alimentar-se tem se tornado uma atividade crítica em todo o mundo. Por um lado, há um considerável aumento nos níveis de obesidade da população e, por outro, um grande contingente de pessoas que ainda sofre de subnutrição. Entre esses dois extremos encontrase uma distribuição desigual de recursos, e uma produção de alimentos cada vez mais industrializada, que acarreta significativos impactos ambientais (UNEP, 2004).

Com o crescimento populacional, estes problemas tendem a se agravar. Diante da necessidade de expansão da produção, agricultura, pecuária e pesca voltam-se para métodos mais intensivos, acarretando uma superexploração dos recursos naturais. Fertilizantes, pesticidas e manipulação genética tornam-se ferramentas cotidianas, agravando ainda mais os impactos ambientais relacionados (UNEP, 2004).

Posto isto, fica patente a necessidade de rever os atuais padrões de produção, distribuição e consumo de alimentos, visando a cenários futuros mais adequados do ponto de vista ambiental, social e econômico.

Neste contexto, o presente artigo apresenta os principais resultados obtidos a partir de um estudo de caso realizado junto à empresa a Whirlpool S.A. ${ }^{1}$. O estudo deu-se a partir de uma parceria entre a empresa e o Núcleo de Design e Sustentabilidade (NDS) da Universidade Federal do Paraná (UFPR)².

Esta parceria teve como objetivo geral o desenvolvimento de proposições de cenários futuros para o cozinhar sustentável. O cenário apresentado caracteriza-se como um sistema produto+serviço (PSS), e foi desenvolvido com base no método MEPPS (Methodology for Product+Service System).

Nas próximas sessões são apresentados o referencial teórico e o método utilizado para o desenvolvimento do PSS, bem como os principais resultados obtidos com o estudo. Na sequência são discutidos estes resultados, e é apresentada a conclusão geral do trabalho.

\section{Referencial Teórico}

Como referência para o delineamento do PSS focado no cozinhar sustentável foram utilizados os resultados do projeto Europeu SusHouse (GREEN; YOUNG, 2000), no qual são descritos possíveis cenários futuros englobando a compra de alimentos, cozimento e alimentação. No estudo é avaliada a viabilidade de cinco cenários, considerando sua aplicação em longo prazo em vários países, incluindo Holanda, Inglaterra, Hungria, entre outros. Os cinco cenários propostos são:

1. Local e verde: os alimentos orgânicos são fornecidos por produtores e pequenos comerciantes locais, e o preparo é feito em casa. São utilizados produtos da estação, e os pratos tradicionais da região são valorizados.

2. Comer Hi-tech: este cenário combina a produção em massa de alimentos de alta qualidade (com a difusão de alimentos geneticamente modificados de baixo impacto ambiental) com tecnologias de armazenamento e cozimento de baixo consumo energético. As casas são equipadas com aparelhos energeticamente eficientes e de alta tecnologia, que permitem o preparo de alimentos mantendo seu valor nutricional e viabilizam pedidos on-line para entrega a domicílio.

\footnotetext{
${ }^{1}$ A Whirlpool S.A, sediada na cidade de Joinville, Santa Catarina, é a maior fabricante de linha branca da América Latina, detentora das marcas Brastemp e Consul.

${ }^{2}$ O NDS-UFPR realiza projetos de pesquisa, atividades de extensão e apoio ao aprendizado acadêmico com foco nas dimensões ambientais, sociais e econômicas da sustentabilidade.
} 
3. Jardim Rural Hi-tech: a maioria das famílias cultiva alimentos em seus jardins utilizando equipamentos de alta tecnologia e baixo impacto ambiental. $\mathrm{O}$ trabalho diário nessas hortas é possível devido à flexibilização dos horários de trabalho e ao trabalho remoto.

4. Centro de Alimentação de Bairro: este cenário combina elementos dos atuais supermercados e restaurantes em um centro de alimentação de bairro, onde as pessoas podem ir para fazer ou comprar refeições e comprar alimentos. Com isto, as cozinhas domésticas podem ter bem poucos equipamentos. Os resíduos são recolhidos e usados para produção de energia local, e os alimentos são cultivados de forma sustentável.

5. Compra Virtual: não há supermercados, os alimentos são comprados via serviços on-line interativos. A comida é entregue nas casas ou em distribuidores locais por meio de transportes de baixo impacto ambiental. As embalagens são reduzidas devido à distribuição a granel, e devolvidas à distribuidora para a coleta e reciclagem (GREEN; YOUNG, 2000).

Buscou-se, a partir desta referência, o desenvolvimento de soluções que apresentassem uma significativa redução dos impactos em relação ao contexto atual, repensando o ato de cozinhar e o modelo de negócios da empresa parceira. O foco mantevese, desta forma, em soluções que promovessem uma mudança radical, caracterizando um alto grau de interferência nos sistemas atuais.

De acordo com Santos (2009), os níveis de interferência do design para sustentabilidade podem ser classificados como (Figura 1):

- Nível 1: melhoria ambiental dos fluxos de produção e consumo

- Nível 2: redesign ambiental do produto

- Nível 3: projeto de novo produto intrinsecamente mais sustentável

- Nível 4: projeto de sistemas produto+serviço

- Nível 5: implementação de novos cenários de consumo "suficiente"

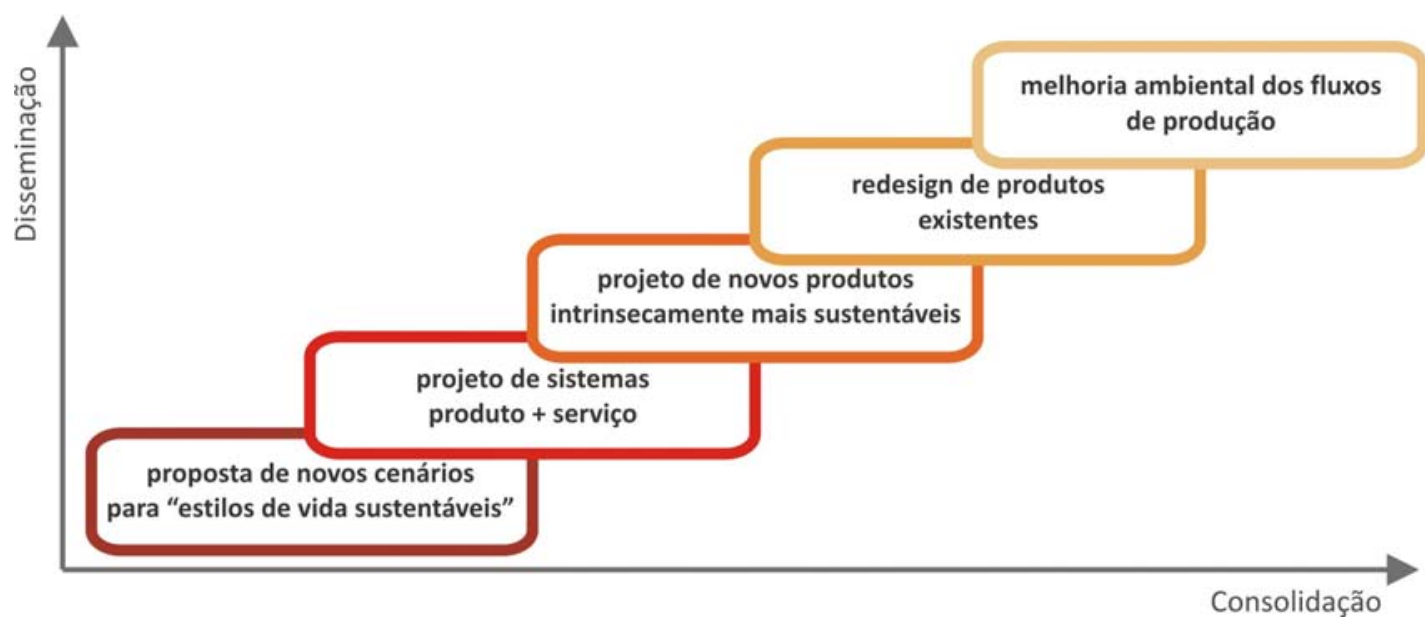

Figura 1 - Níveis de interferência do design para sustentabilidade Fonte: Adaptado de SANTOS (2009) 
Segundo esta classificação, o nível inicial relaciona-se à melhoria ambiental dos fluxos de produção, visando evitar a poluição e os desperdícios. Ainda que viabilizem avanços no desempenho ambiental, as interferências ocorridas neste nível são restritas em seus impactos, principalmente por terem pouca influência nos padrões de consumo (SANTOS, 2009).

O segundo nível envolve o redesign ambiental de produtos, melhorando sua ecoeficiência. Neste nível, um produto existente é ambientalmente readequado, por meio, por exemplo, da substituição de materiais e de alterações que permitam uma maior eficiência no consumo de matéria-prima e energia, ao longo de seu ciclo de vida (MANZINI; VEZZOLI, 2002).

O terceiro nível diz respeito ao projeto de novos produtos ou serviços, priorizando soluções ambientalmente mais favoráveis. Neste nível, procura-se melhorar o desempenho do produto em todas as etapas do ciclo de vida, por meio da aplicação de estratégias como: utilização de recursos de baixo impacto ambiental, otimização do ciclo de vida do produto, extensão da vida dos materiais e facilitação da montagem e desmontagem (MANZINI; VEZZOLI, 2002).

O quarto nível engloba o projeto de novos sistemas de produto+serviço intrinsecamente sustentáveis, visando oferecer soluções inovadoras e radicalmente favoráveis ao meio ambiente. Neste nível se dá uma mudança de foco, que passa do consumo de bens para o consumo de serviços, buscando soluções sistêmicas (MONT, 2000). Desta forma, torna-se possível promover a satisfação dos usuários desmaterializando (total ou parcialmente) o consumo. A aplicação destes sistemas demanda mudanças bastante significativas na estrutura das empresas e nos estilos de vida dos usuários. Envolve ainda consideráveis transformações culturais e comportamentais, uma vez que interfere na noção de "posse" dos produtos (SANTOS, 2009).

Por fim, o último nível abrange a proposta de novos cenários que correspondam a "estilos de vida sustentáveis", com o objetivo de promover novos critérios de qualidade que sejam sustentáveis, aceitáveis e atraentes (MANZINI; VEZZOLI, 2002). Há grandes desafios para a aplicação deste nível, que demanda mudanças profundas na dinâmica das estruturas sociais, com a adoção de valores culturais radicalmente diferentes daqueles vigentes no atual paradigma (SANTOS, 2009).

O projeto aqui relatado enquadra-se, portanto, no quarto nível, referente ao projeto de sistemas produto+serviço (PSS). A implantação de sistemas desta natureza é atualmente considerada uma alternativa válida para aumentar a competitividade das empresas, promovendo, ao mesmo tempo, a redução de impactos ambientais (TUKKER, 2004).

O Sistema Produto+Serviço é uma das ferramentas do design para a sustentabilidade e possui como objetivo reduzir o conteúdo material por unidade de satisfação. Segundo a United Nations Environment Programme (UNEP, 2002, p.4)

\footnotetext{
Sistemas Produto+Serviço podem ser definidos como o resultado de uma estratégia de inovação, redirecionando o foco de negócios do design e venda de produtos físicos, para a venda de sistemas de produtos e serviços que são conjuntamente capazes de atender integralmente demandas específicas dos clientes.
}

Além de focar na utilização em vez da aquisição de produtos, o PSS também possui o objetivo de integrar ao ciclo de vida do produto diversos serviços, como por exemplo: manutenção, troca, locação, upgrade, remanufatura, coleta e disposição final. Deste modo, 
objetiva-se otimizar o uso do produto/serviço oferecido, reduzindo ao máximo a quantidade de resíduos em fim de vida.

O PSS também se propõe a efetuar a análise da cadeia de fornecedores que interagem em todas as fases do processo, buscando estabelecer parcerias que possibilitem a construção de uma rede de stakeholders que possuam interesse em atuar conjuntamente no processo de gestão dos produtos/serviços oferecidos.

Para Tukker (2004), este tipo de sistema permite às empresas agregar valor às suas ofertas e aumentar sua competitividade, uma vez que:

- Permitem atender às necessidades dos clientes de forma integrada e personalizada;

- Facilitam a construção de relacionamentos exclusivos com os clientes, aumentando a fidelização ;

- Promovem maior agilidade no processo de inovação, uma vez que a empresa consegue acompanhar de forma mais próxima as necessidades dos clientes (TUKKER, 2004).

Desta forma, considera-se relevante a realização de estudos focados no desenvolvimento e implementação de PSS, visando à disseminação destes conceitos, ao teste de métodos específicos para este fim e ao fornecimento de parâmetros para empresas que planejam fazer a transição da venda de produtos para a oferta de soluções sistêmicas.

\section{Método}

Foi realizado um estudo de caso com a empresa Whirlpool que utilizou como base o MEPSS - Metodologia para Sistema Produto+Serviço proposto por Halen, Vezzoli e Wimmer (2005) e as proposições de Vezzoli (2010) para o design de sistemas para a sustentabilidade. O objetivo desta metodologia é desenvolver soluções sistêmicas alinhadas com os objetivos de negócio das empresas, satisfazendo os anseios e as necessidades dos clientes e ao mesmo tempo avaliando os seus impactos econômicos, ambientais e sociais.

A estrutura metodológica do MEPSS consiste de cinco fases principais:

Fase I - Análise estratégica: busca-se construir um conhecimento aprofundado e sistemático dos mercados da empresa, organização, produção e cadeia de valor.

Nesta fase são utilizadas duas ferramentas: SDO (Sustainability Design-Orienting toolkit) e Mapa do sistema.

O SDO é utilizado para orientar o processo de design para soluções sustentáveis. Segundo Vezzoli (2010) o propósito do SDO é:

- Definir as prioridades de projeto para as três dimensões da sustentabilidade (ambiental, social e econômica), através da análise do sistema existente por meio de checklists;

- Estimular a geração de idéias voltadas ao desenvolvimento de sistemas sustentáveis, tendo como referência as diretrizes de projeto apresentadas pela ferramenta;

- Analisar as melhorias/prejuízos relativos às três dimensões da sustentabilidade, comparando o sistema proposto com o sistema atual através de diagramas de radar. 
O Mapa do Sistema é uma ferramenta gráfica que ajuda a visualizar os principais atores do sistema e as suas relações (fluxos de material, informação, dinheiro e trabalho). Para Vezzoli (2010) o Mapa do Sistema é uma ferramenta de suporte para:

- Projetar, pois estrutura o pensamento e facilita a resolução de problemas;

- Coprojetar, porque utiliza um padrão de linguagem que pode ser compartilhado facilitando assim o diálogo dos atores envolvidos;

- Comunicar, pois facilita a visualização da solução projetada.

Fase II - Exploração de oportunidades: procura-se explorar possíveis inovações para o sistema atual. O objetivo não é apenas chegar a melhorias incrementais de produtos e serviços, mas criar possíveis inovações a nível sistêmico.

As ferramentas utilizadas nesta etapa são: Mapa do Sistema, SDO e Diagrama de Polaridade.

Como as ferramentas Mapa do Sistema e SDO já foram apresentadas anteriormente, abaixo será apresentada apenas o Diagrama de Polaridade.

O Diagrama de Polaridade tem como objetivo dar suporte à construção de cenários. De acordo com Vezzoli (2010) esta ferramenta ajuda na geração de idéias e facilita a sua organização e apresentação. O diagrama é construído a partir do cruzamento de duas idéias/conceitos opostos. Um deles é geralmente relacionado com o tipo de solução a oferecer (serviço total versus plataforma) e o outro está relacionado com o nível de participação dos stakeholders (baixo envolvimento versus alto envolvimento).

Fase III - Desenvolvimento do conceito de PSS: busca-se descobrir como as oportunidades detectadas na análise e nos cenários podem ser utilizadas com sucesso. Esta fase passa de uma descrição geral e visual para uma versão mais precisa, avaliando e selecionando a versão mais promissora antes de colocá-la no mercado.

As ferramentas utilizadas nesta etapa são: Mapa do Sistema, SDO, Diagrama de Polaridade e Story-board. Abaixo a descrição da ferramenta Story-board.

O Story-board é a tradução de um evento utilizando uma sequência de imagens estáticas (ilustrações ou fotografias) associadas a um texto que funciona como complemento explicativo das imagens. Para Manzini e Jégou (2003) o conteúdo da narrativa deve considerar a interação entre o usuário e o sistema, descrevendo a experiência do ponto de vista do usuário e a interação entre os diversos atores na produção e distribuição da oferta. Basicamente o story-board descreve visualmente as diversas interações que acontecem ao longo do ciclo de vida do produto, da produção ao descarte.

Fase IV - Desenvolvimento do PSS escolhido: busca identificar qual o parâmetro de design com maior possibilidade de sucesso, e desenvolvê-lo. O objetivo principal desta fase é detalhar cada dimensão do sistema e elaborar as especificações necessárias para tornar possível a sua implementação.

As ferramentas utilizadas nesta etapa são: SDO, Diagrama de Polaridade, Storyboard e Story Spot. Abaixo a descrição da ferramenta Story Spot.

O Story Spot descreve sucinta e efetivamente, os elementos mais importantes do sistema, ou seja, foca em apenas algumas interações específicas. Para Vezzoli (2010) o Storyspot deve apresentar os seguintes elementos-chaves: as interações mais relevantes do cliente com a oferta fornecida pelo sistema, as interações-chave dos diversos atores durante a produção e o fornecimento da oferta e de que forma a solução projetada alcança as metas definidas. 
Fase V - Implementação do PSS: procura-se identificar quais são os instrumentos condutores do processo de implementação e como controlar o comportamento do sistema proposto.

\section{Projeto Monno: Resultados e análise}

\section{Análise Estratégica}

O desenvolvimento do PSS se iniciou com a caracterização da empresa e do público-alvo.

A Whirlpool S.A. é formada por meio da reorganização societária das marcas brasileiras Brastemp e Consul (Multibrás S.A. Eletrodomésticos), e Embraco (Empresa Brasileira de Compressores S.A.). Atua no setor industrial de linha branca - fogões, freezers horizontais e verticais, refrigeradores, lavadoras de roupa, secadoras, lava-louças, microondas, fornos elétricos, condicionadores e depuradores de ar, coifas e climatizadores (WHIRLPOOL LATIN AMÉRICA, 2010a).

A maior fábrica de produtos de refrigeração da Whirlpool Corporation está localizada no município de Joinville. Esta fábrica é certificada pelo Sistema de Gestão Integrada (SGI), que a credencia com a ISO 9001 (Qualidade), ISO 14001 (Meio Ambiente), OHSAS 18001 e IECQ - QC 080000 HSPM (Saúde e Segurança) (WHIRLPOOL LATIN AMÉRICA, 2010b). A parceria entre a UFPR e a unidade de Joinville foi escolhida como objeto de estudo pela sua localização, por apresentar em sua estrutura um departamento de design, bem como contribuir em grande parte ao desenvolvimento de produtos para a região Whirlpool América Latina, que lança anualmente 150 novos produtos (WHIRLPOOL LATIN AMÉRICA, 2010a).

O estudo de caso foi realizado com a empresa Brastemp por ser sinônimo de qualidade e inovação no setor de eletrodoméstico. Esta resume os seus principais atributos com as seguintes palavras: surpreendente, inovadora, elegante com estilo e irreverente (WHIRLPOOL LATIN AMÉRICA, 2010a).

Segundo a empresa seus clientes (ibidem):

- Gostam de ser surpreendidos (buscam produtos que saem do lugar comum)

- São curiosos e dinâmicos (gostam de novidades e de descobrir novas soluções)

- Permitem-se experiências que extrapolam o funcional

- São ligados no mundo (buscam referências do que acontece ao seu redor)

- São visuais e vaidosos (diferenciam-se através de detalhes)

Posteriormente iniciou-se um estudo preliminar para determinar o mapa do sistema atual da empresa (Figura 1). Este teve com objetivo fornecer uma visão geral do sistema e seus fluxos (financeiro, de trabalho, de informações e de materiais), bem como das interações entre os principais atores envolvidos. 


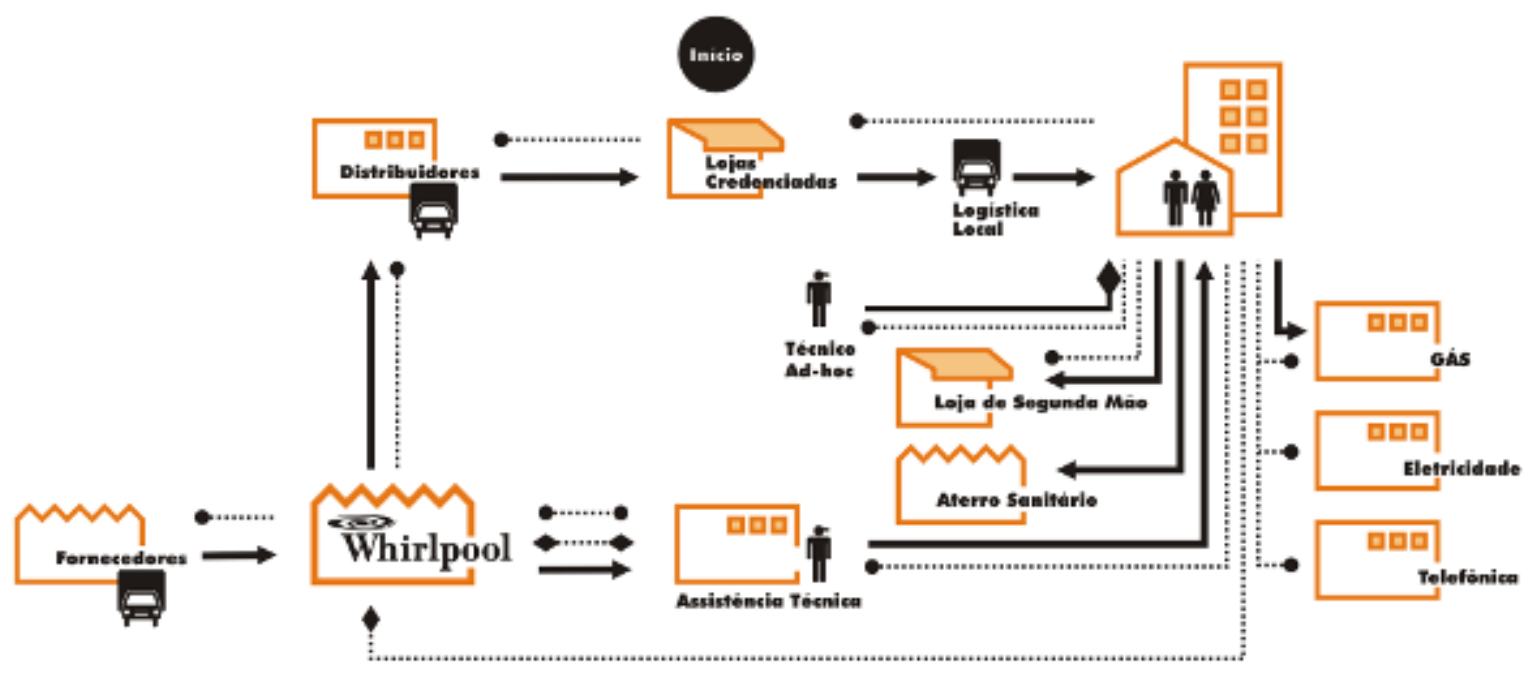

Figura 2 - Sistema atual da empresa

Buscou-se processar estas informações para conduzir a geração de soluções potencialmente sustentáveis. Nesse contexto, foi definida a unidade de satisfação a ser atendida pelo sistema: "Cozinhar de forma sustentável".

\section{Exploração das Oportunidades}

A geração de um grupo de idéias potencialmente sustentáveis para o atendimento da unidade de satisfação do sistema foi realizada por meio da ferramenta diagrama de polaridade (Figura 2).

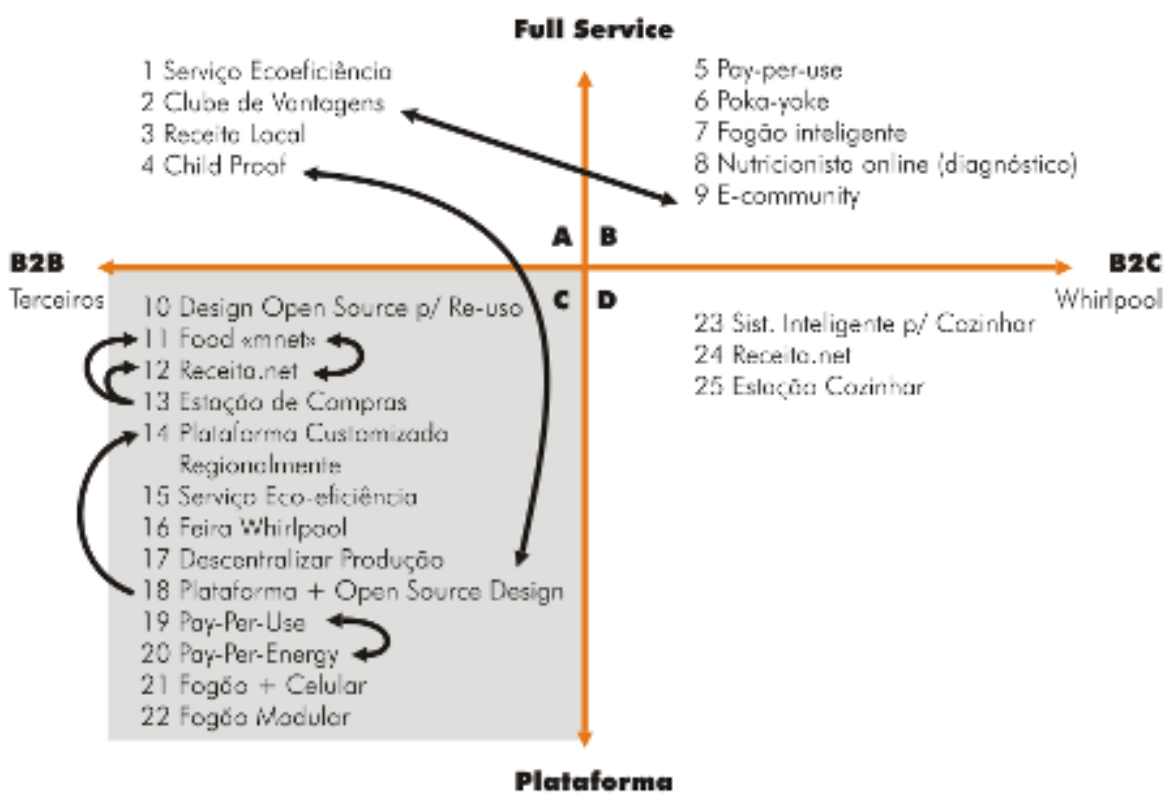

Figura 3 - Diagrama de Polaridade 
A partir da análise do sistema atual determinou-se que o quadrante $C$ (em destaque) apresentou soluções factíveis ao desenvolvimento pela empresa de novas soluções para o atendimento das necessidades do usuário.

As soluções que possuem estreita relação no atendimento da unidade de satisfação (similares ou complementares) foram ligadas no diagrama de polaridade para facilitar formação de conceitos para o sistema. Ao final dessa etapa, foram selecionados 2 conceitos complementares que reúnem as melhores soluções apresentadas no diagrama de polaridade (Figura 3).

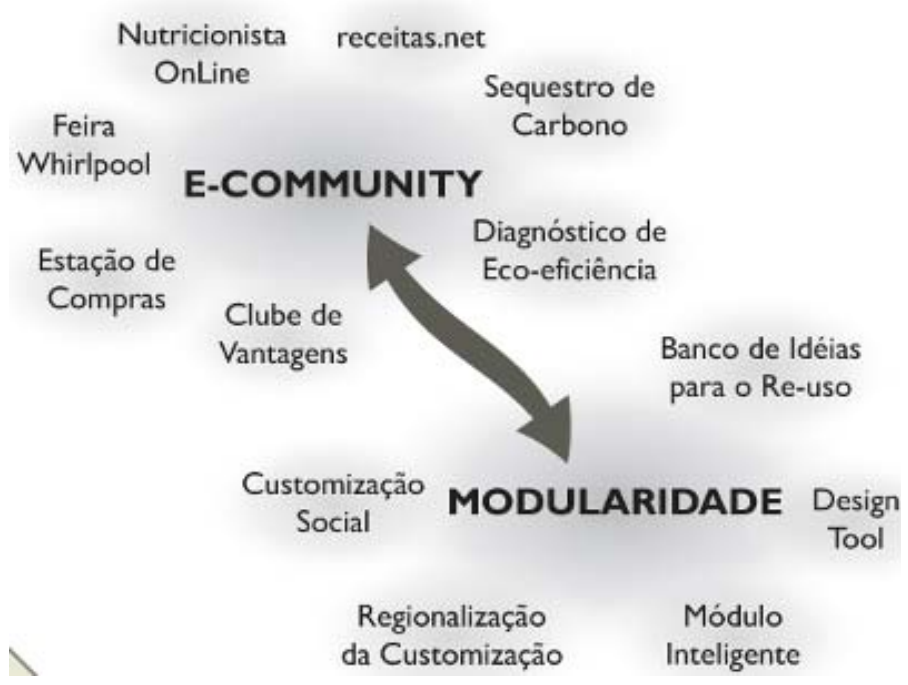

Figura 4 - Geração de Conceitos de Sistema

O conceito Modularidade está diretamente relacionado ao produto, enquanto, o conceito E-Community refere-se a relação do usuário com os serviços ofertados pelo sistema.

Desenvolvimento dos Conceitos de PSS

O conceito E-Community consiste em uma rede de serviços ofertados ao usuário que Ihe permite ter acesso aos seguintes benefícios: Nutricionista Online: orientações de alimentação saudável; Receitas.net: Portal de receitas; Feira Whirlpool: promover a interação com fornecedores locais; Estação de Compras: rotas sustentáveis de compra; Clube de Vantagens: promover o comportamento sustentável através de um sistema de pontuação; Sequestro de Carbono: venda do crédito de carbono para a empresa; Diagnóstico de Eco-eficiência: Serviço de avaliação da ecoeficiência dos produtos Whirlpool.

O conceito Modularidade consiste na melhoria da funcionalidade do produto por meio do uso de peças componíveis. Este conceito é viabilizado no sistema pelas seguintes proposições: Banco de Idéias para o Re-uso: disponibilizar idéias de segundo uso para modificação das peças descartadas; Design Tool: disponibilizar ferramentas de padronização de projeto (BIM - Building Information Manufacture); Módulo Inteligente: permitir a criação de módulos com funcionalidades diversas; Regionalização da Customização: possibilitar o desenvolvimento e a fabricação de módulos, por fabricantes regionais; Customização Social: possibilitar o desenvolvimento de módulos para usuários com necessidades especiais. 
Desenvolvimento do PSS Escolhido

Para visualizar as interações entre os principais atores do sistema proposto, foi desenvolvido um novo system map.

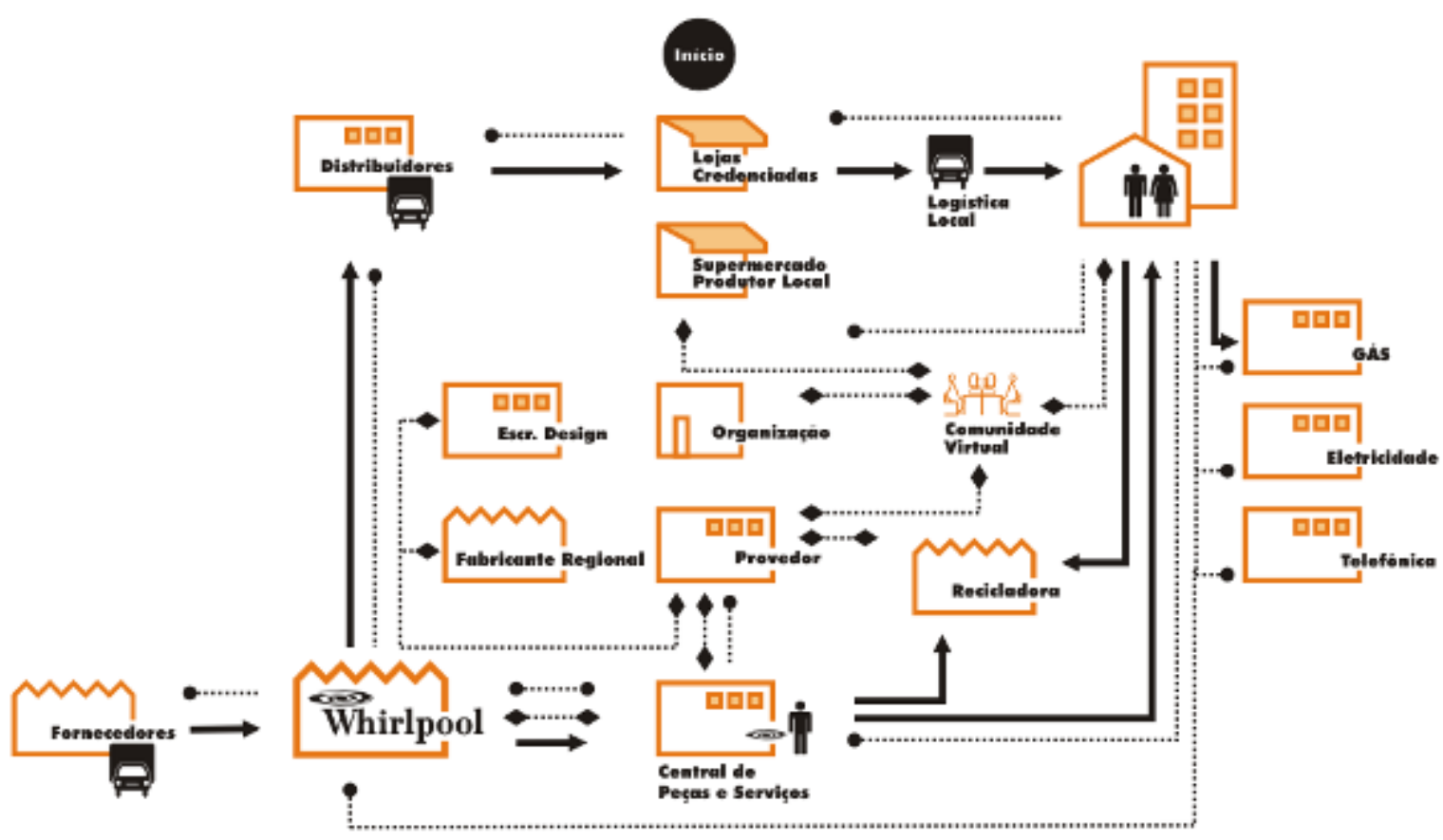

Figura 5 - Mapa do Sistema Proposto

Um estudo preliminar do storyboard foi desenvolvido para a visualização do funcionamento dos dois conceitos propostos no sistema ao longo do ciclo de vida do produto. Nele constavam interações básicas para um cenário geral de uso, mostrando as interações de stakeholders com o consumidor do momento de decisão da aquisição até o descarte. Esta etapa do processo permitiu identificar a atuação de cada stakeholder na linha do tempo.

Com a proposição de um novo sistema, a definição de requisitos voltados para a sustentabilidade foi realizada por meio do SDO - Sustainability Design Orienting Toolkit com a definição de prioridades dentro de cada uma das principais dimensões da sustentabilidade (ambiental, sócioética e econômica). Para o desenvolvimento dos conceitos foram levados em conta apenas os requisitos que apresentavam alta e média prioridade, conforme apresentado nos radares a seguir: 


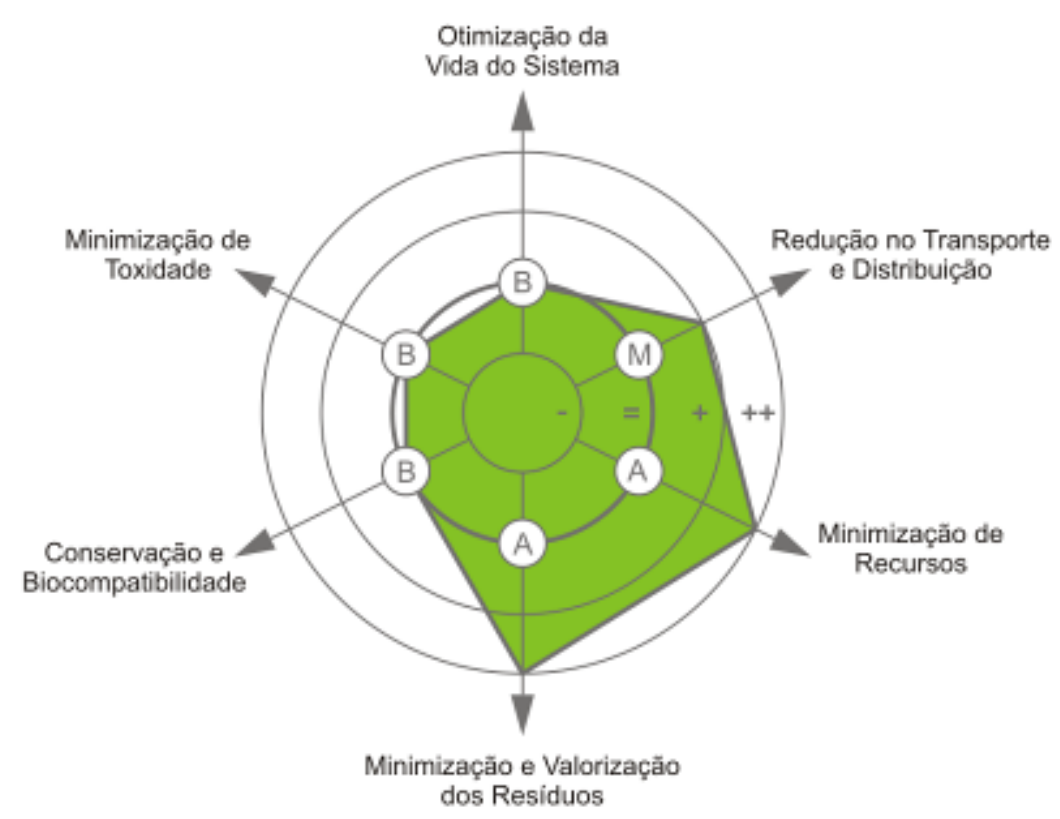

Figura 6 - Dimensão Ambiental do Sistema Proposto

Fonte: SDO (2010)

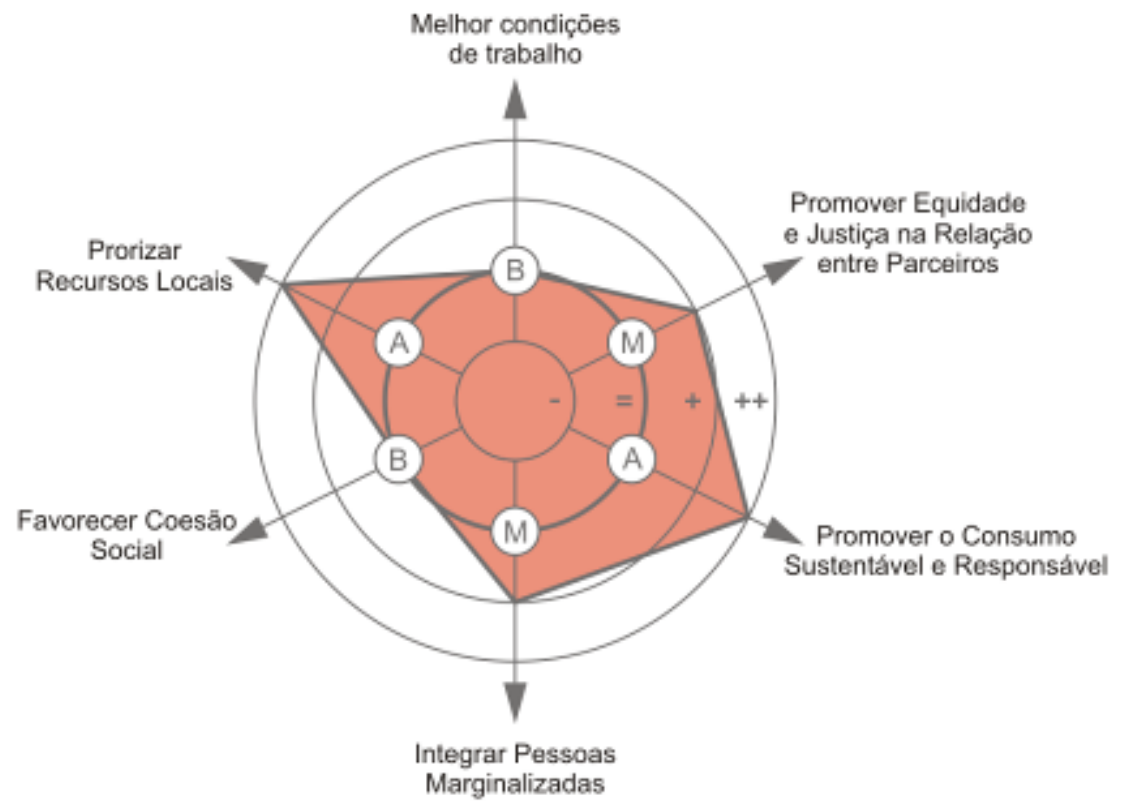

Figura 7 - Dimensão Socioética do Sistema Proposto Fonte: SDO (2010) 


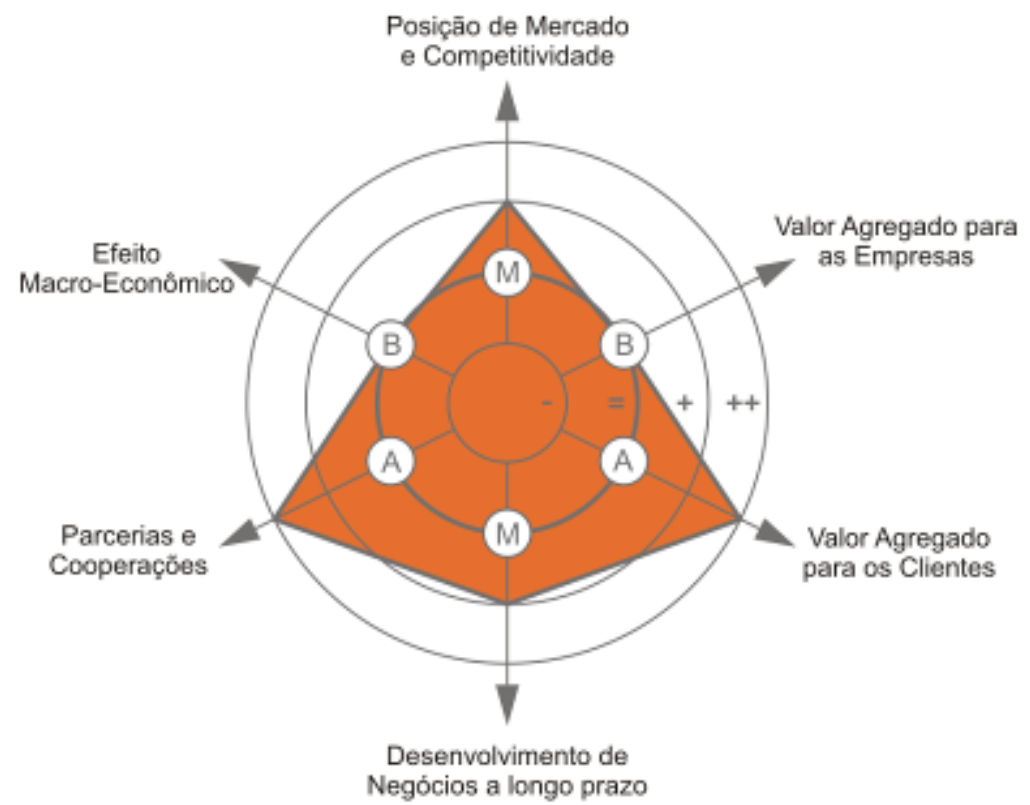

Figura 8 - Dimensão Econômica do Sistema Proposto

Fonte: SDO (2010)

Para cada um desses requisitos foram propostas soluções que deveriam ser integradas nos serviços e produtos finais. Com estas proposições a equipe iniciou o processo criativo, utilizando uma adaptação da técnica 6:3:5. As idéias geradas foram agrupadas por semelhança e selecionadas para a alternativa final. Por meio da combinação dessas idéias foi desenvolvido e refinado o produto, chegando ao estágio de modelagem digital.

Retomando os stakeholders definidos no inicio do processo projetual, um storyboard completo foi criado, mostrando paralelamente as ações de todos os agentes envolvidos no sistema, e incluindo visões de mais de um cenário possível. Ao todo foram apresentados 13 stakeholders em 29 ações do ciclo de vida dos produtos e serviços componentes do sistema, divididas em etapas de identificação de oportunidade, projeto, distribuição/instalação, uso e descarte (Figura 8). 


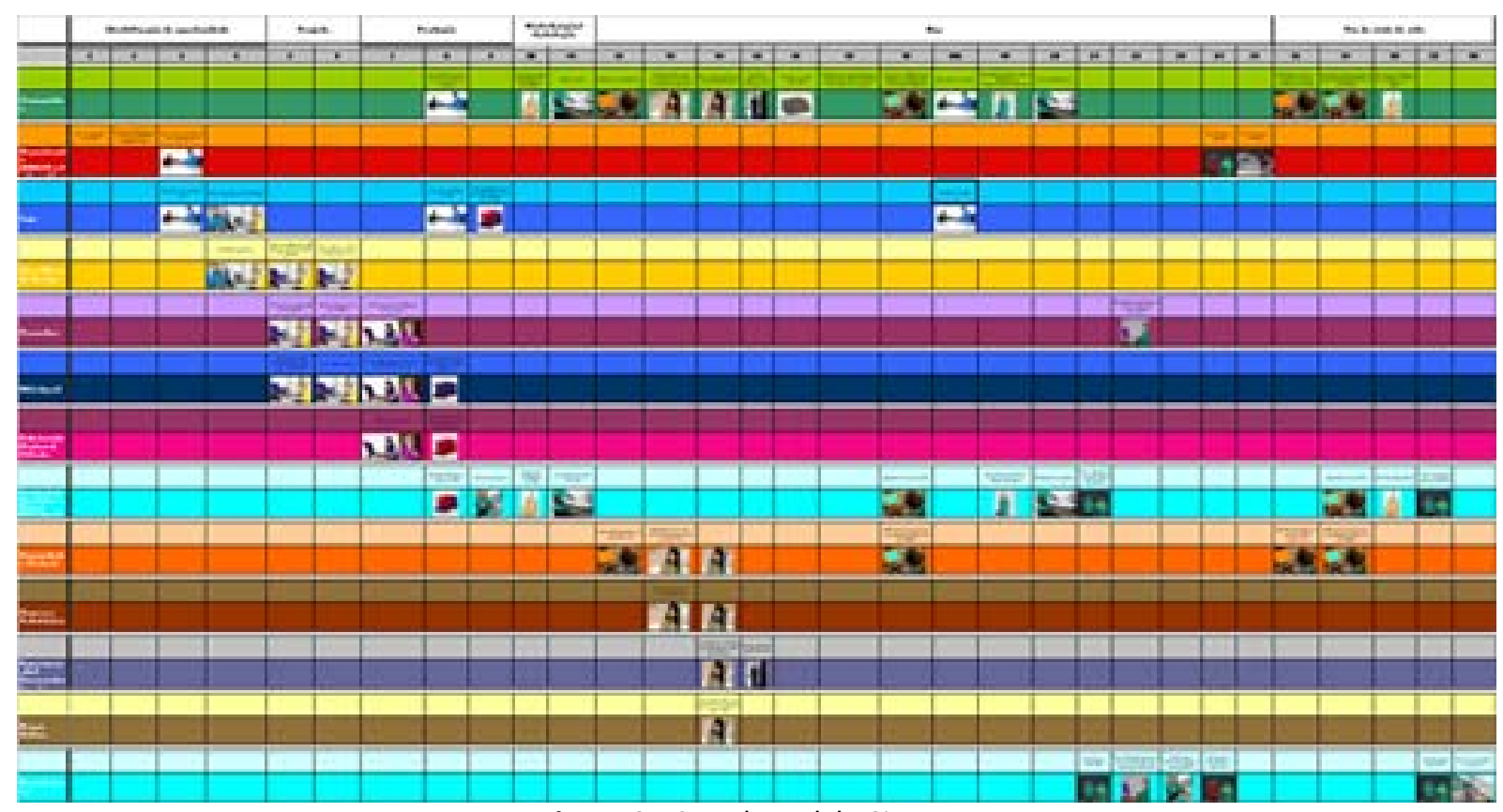

Figura 9 - Storyboard do Sistema

Uma versão do storyboard para a visualização apenas da inovação para o consumidor foi gerada (Figura 9), e também um Story Spot (Figura 10) com as principais relações entre stakeholders e a resposta para os requisitos sustentáveis encontrados na fase de conceituação do projeto através do SDO.

\begin{tabular}{|c|c|c|c|c|c|c|}
\hline 10 & 11 & 12 & 13 & 14 & 15 & 16 \\
\hline $\begin{array}{l}\text { Recebe o fogão da } \\
\text { Central de Serviços } \\
\text { e Pesas Whirpool }\end{array}$ & Fogāo instalasdo & $\begin{array}{c}\text { Cadastro na comuridade } \\
\text { vitual }\end{array}$ & $\begin{array}{l}\text { Decide cozinhar: acessa a } \\
\text { communidade virtud atraves do } \\
\text { telefone para escolher receta }\end{array}$ & $\begin{array}{l}\text { Encomenda ingredentes } \\
\text { do fornecedor bccal via } \\
\text { intermet }\end{array}$ & $\begin{array}{l}\text { Reccebe ors } \\
\text { ingredientess } \\
\text { realza a rocta } \\
\text { econbemica }\end{array}$ & $\begin{array}{c}\text { Cozinha com os módulos } \\
\text { especialzzados }\end{array}$ \\
\hline & & $=7$ 다 & & & & \\
\hline
\end{tabular}

Figura 10 - Recorte da linha de interação do consumidor no sistema 


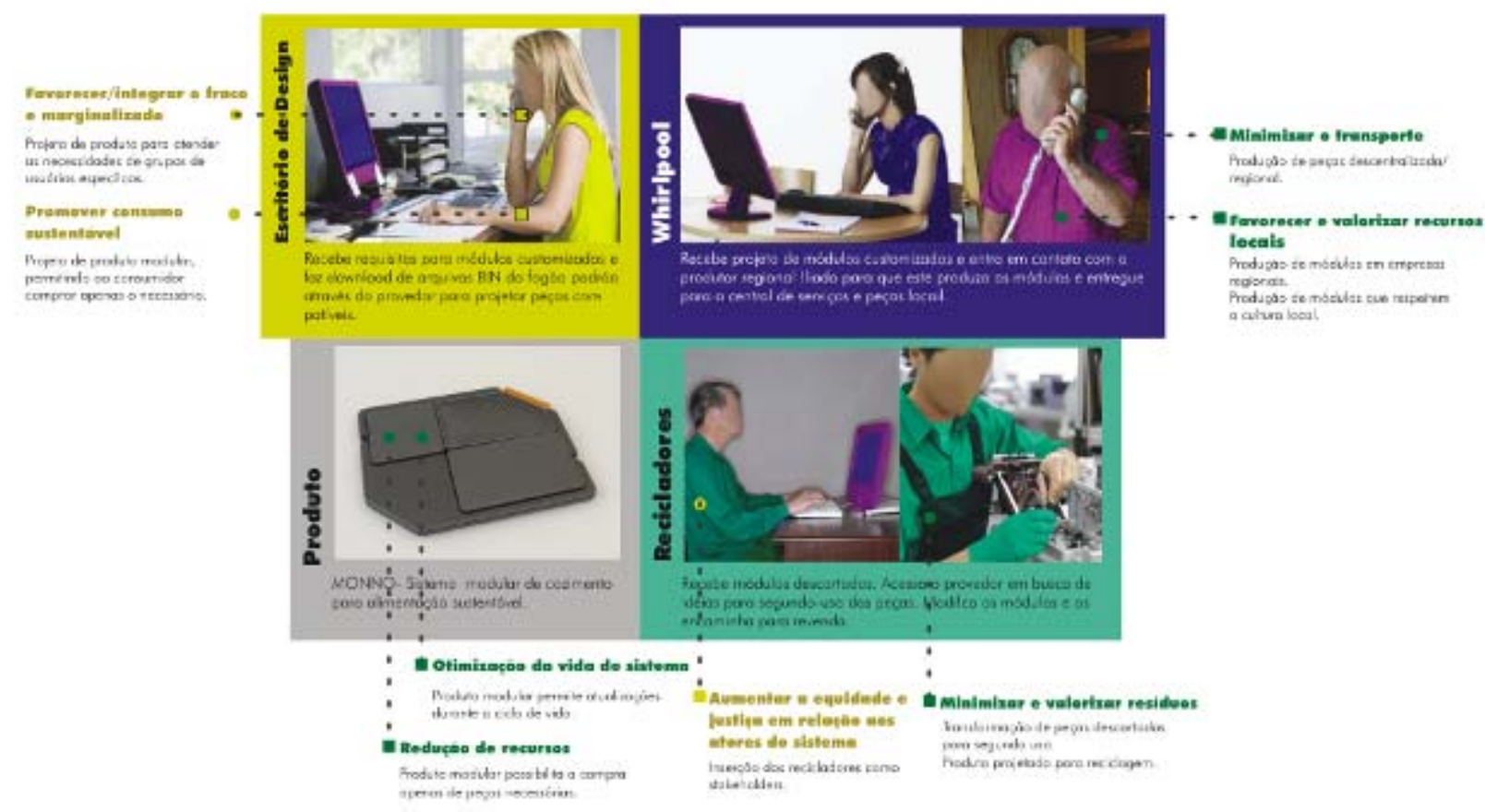

Figura 11 - Interaction Story Spot do sistema proposto

Foi desenvolvido um produto com os conceitos E-community e Modularidade para atender o sistema proposto. O produto possibilita a criação de módulos para atender necessidades específicas do usuário (por exemplo, aspectos culturais regionais) e apresenta uma tela LCD para acesso as suas funções e a comunidade virtual. Suas características permitem a descentralização da produção de seus componentes e atualização progressiva evitando seu descarte prematuro.

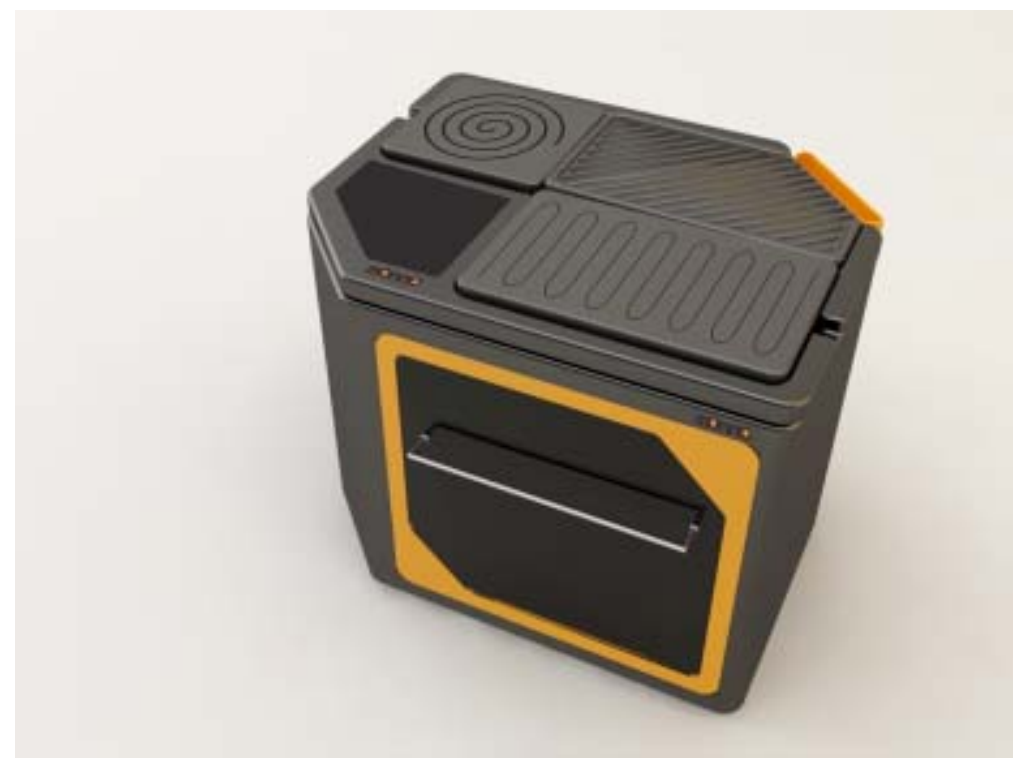

Figura 12 - Fogão Modular Monno 


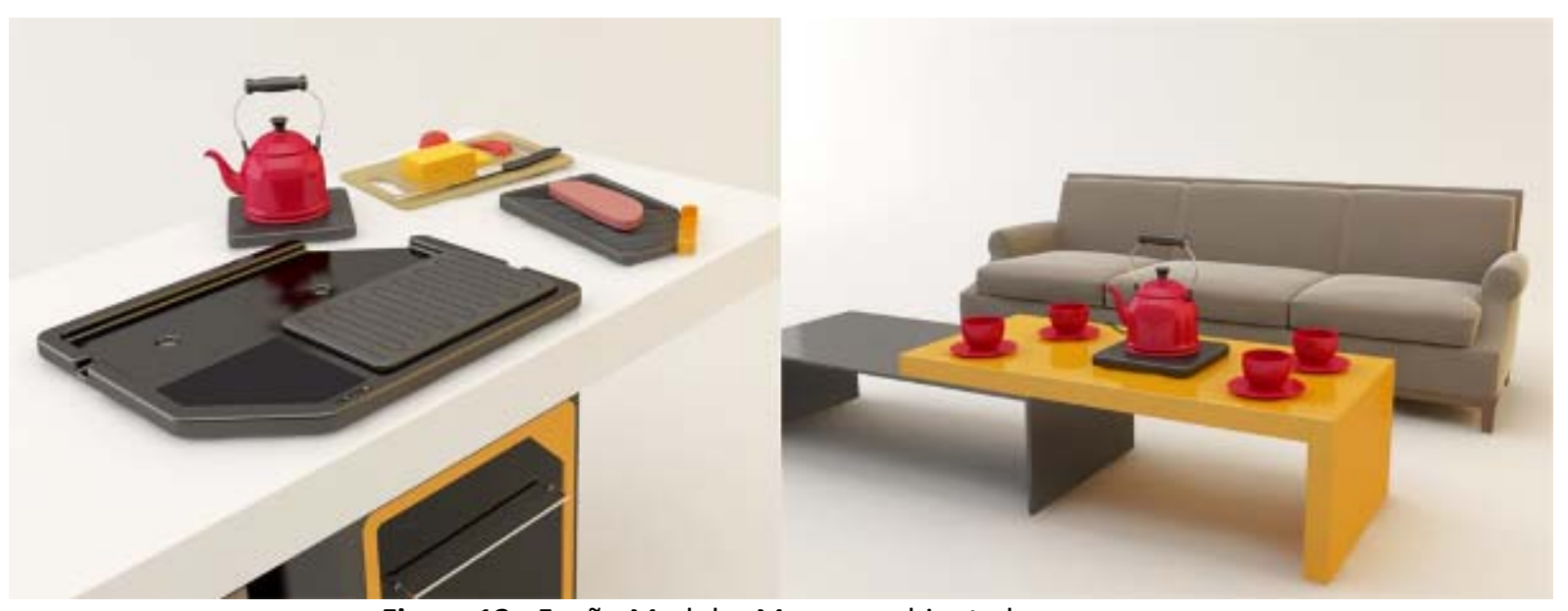

Figura 13 - Fogão Modular Monno ambientado

Considerações Finais

Diante da evidência da inter-relação das crises ambientais, econômicas e sócioculturais, torna-se cada vez mais claro que o cenário da "redesign do existente" não é suficiente para o desenvolvimento de soluções verdadeiramente sustentáveis. (MANZINI, 2002). Para atender esse novo cenário torna-se necessário intensificar o desenvolvimento de projetos dedicados ao atendimento da interferência do design para a sustentabilidade a nível estratégico.

Como resultado, a solução proposta apresenta produtos e serviços que reduzem o conteúdo material necessário para o atendimento da unidade de satisfação em comparação com o sistema existente. Integra serviços que melhoram o desempenho ambiental e socioético do produto ao longo de seu ciclo de vida. Nesse contexto, os resultados apresentados corroboram com as características de PSS estudadas inicialmente.

O PSS desenvolvido englobou características de 4 dos 5 cenários do Europeu SusHouse apresentados anteriormente. Os cenários incluídos foram o Local e Verde, Comer Hi-tech, Centro de Alimentação do Bairro e Compra Virtual, por integrar os atores mais relevantes, atendendo a unidade de satisfação do sistema proposto. O cenário Jardim Rural Hi-tech não foi incluído, pois não representava os hábitos do público alvo.

Outro fator que se destaca no presente estudo diz respeitos às dificuldades no desenvolvimento do PSS por meio da metodologia MEPSS. Nesse sentido, os fatores mais influentes no resultado do projeto foram a limitação dos dados coletadas durante a fase de análise estratégica, complexidade das ferramentas utilizadas e o tempo necessário para o desenvolvimento do projeto.

O design tem mudado seu campo de ação, atuando além da concepção de artefatos, para o desenvolvimento de soluções sistêmicas, atuando a um nível mais estratégico. Essa expansão do seu campo de atuação traz novas oportunidades de desenvolvimento de soluções com maior desempenho ambiental e socioético, destacando o estudo do PSS como um meio prático de atingir melhores resultados projetuais. 


\section{Referências}

GREEN, Ken; YOUNG, William. Shopping, cooking and eating function: The Netherlands: Delft University of Technology, 2000.

HALEN, Cees Van. VEZZOLI, Carlo;. WIMMER, Robert. Methodology for product service system innovation: how to implement clean, clever and competitive strategies in European industries. Royal Van Gorcum. Assen, The Netherlands, 2005.

MANZINI, Ezio; JEGOU, François. Sustainable everyday: scenarios of urban life. Milão: Edizione Ambiente, 2003.

. O desenvolvimento de produtos sustentáveis: os requisitos ambientais dos produtos industriais. São Paulo: EDUSP, 2002.

MONT, Oksana. Product service-systems. Sweden: Lund University, 2000. (IIIEE Reports)

SANTOS, Aguinaldo dos. Níveis de maturidade do design sustentável na dimensão ambiental. Cadernos de Estudos Avançados em Design, Barbacena, v. 3, p. 13-26, 2009.

UNITED NATIONS ENVIRONMENT PROGRAMME - UNEP. Food: feed the world without starving the planet. In: . Resource kit on sustainable production and consumption. Paris, 2004. Disponível em: <http://www.unep.org/pdf/sc/sc_resourcekit.pdf>. Acesso em: 6 dez. 2010.

. The role of product-service systems in a sustainable society. Paris, 2002.

VEZZOLI, Carlo. Design de Sistemas para a Sustentabilidade. Salvador: EDUFBA, 2010.

WHIRLPOOL LATIN AMÉRICA. Apresentação institucional. Joinville, 2010a. Disponível em: <http://www.whirlpool.com.br>. Acesso em: 10 out. 2010.

.Whirlpool: site institucional. Joinville, 2010b. Disponível em:

<http://www.whirlpool.com.br>. Acesso em: 10 out. 2010. 